\title{
Structural and Magnetic Properties of "Thick" Co-rich Microwires Produced by the Ulitovsky-Taylor Method
}

\author{
E.E. Shalygina ${ }^{1, *}$, A.N. Shalygin ${ }^{1}$, A.M. Kharlamova ${ }^{1}$, V.V. Molokamov ${ }^{2}$, N.V. Umnova ${ }^{2}$, P.P. Umnov ${ }^{2}$ \\ ${ }^{1}$ Faculty of Physics, Moscow State University named M.B. Lomonosov, 119991, Moscow, Russia \\ ${ }^{2}$ Baikov Institute of Metallurgy and Materials Science, 119991 Moscow, Russia \\ *Corresponding Author: shal@magn.ru
}

Copyright (C) 2014 Horizon Research Publishing All rights reserved.

\begin{abstract}
The structural and magnetic properties of as-cast and annealed Co-rich microwires in a glass shell with the diameter of $D=125 \mu \mathrm{m}$ and the diameter of the amorphous metallic core of $d=50-90 \mu \mathrm{m}$, produced by the modernized Ulitovsky-Taylor method, have been investigated. The saturation field $H_{S}$ and the coercive force $H_{C}$ of the samples were found to depend on annealing temperature. The near-surface values of $H_{S}$ and $H_{C}$ were discovered to be larger than the bulk ones. The obtained experimental data were explained by the structural features of the microwires under study.
\end{abstract}

Keywords Magnetic characteristics, Microwires, Structural properties, Ulitovsky-Taylor method.

\section{Introduction}

Although amorphous magnetic materials were discovered more 50 years ago, the interest to studying their structural, magnetic and kinetic properties remains up to now. This fact is cased by the possibility of wide use of amorphous materials in modern micro- and nanoelectronics with relatively low cost of their production. Amorphous magnetic materials have been used for producing magnetic heads, magnetic screens, transformer cores, magnetostriction vibrators, delay lines, etc. [1-3]. The area of the amorphous alloys applications expands continuously. Amorphous materials, prepared as microwires, deserve special attention. In particular, the revealed unique magnetic properties of amorphous microwires were caused their wide application in practice for manufacturing high sensitive sensors of magnetic fields, stresses, low pressures, strains, etc., and also new types of coding devices [4-12].

There are several methods of producing microwires. At the present time, one of the widely used methods is the method of pulling of fusion from a quartz ampoule through the calibrated opening in cooling liquid [13-15]. In this case microwires have no glass cover. The metallic microwires with glass shell were produced by means of the method, proposed by G.F. Taylor in 1924 [16]. Later, this method was improved by A.V. Ulitovsky [17, 18]. In this case amorphous microwires had the metallic core diameter no more than 40-45 microns and external glass layer about 0.5-15.0 microns.

Recently, a new type of wires, namely, "thick" amorphous wires with the metallic core from 50 up to 160 microns in diameter were prepared from soft magnetic $\mathrm{Fe}-\mathrm{Co}$ alloys using the modernized Ulitovsky-Taylor method [19]. The study of the physical properties of the new type of microwires deserved attention as from the scientific and practical points of view. The first experimental data of such investigations had been published in [20].

In this paper we present the results of the detailed investigation of the mechanical, elastic and magnetic characteristics of the as-cast and annealed thick Co-rich microwires, produced by the modernized Ulitovsky-Taylor method.

\section{Materials and Methods}

The $\mathrm{Co}_{69} \mathrm{Fe}_{4} \mathrm{Cr}_{4} \mathrm{Si}_{12} \mathrm{~B}_{11}$ microwires were produced by the modernized Ulitovsky-Taylor method by using alloys, prepared from components with purity higher than $99.8 \%$ [19]. To increase the alloy purity during preparing the melt, the pumping, the refinement with use helium-hydrogen mix and also homogenization of the fusion were performed. The ingot part was used for preparing rapidly quenched rods from which the microwires with glass shell were produced with the help of the Ulitovsky-Taylor method.

The microwires under study had the diameter $D=125$ microns and the diameter of the amorphous metallic core $d$ $=90$ microns. The $\mathrm{Co}_{69} \mathrm{Fe}_{4} \mathrm{Cr}_{4} \mathrm{Si}_{12} \mathrm{~B}_{11}$ microwire was annealed in a muffle furnace at the annealing temperatures $T=200-430^{\circ} \mathrm{C}$ for $10 \mathrm{~min}$. The influence of the glass shell on the microwire properties was studied by its mechanical removal.

The amorphous state of the as-cast microwires was checked with the help of X-ray diffraction (XRD).

The plasticity level of the microwires was estimated by its 
ability to be tied into a knot without fracture. In this case the opposite ends of a microwire, preliminary, tied in knot, were tightened with a speed of $0.02 \mathrm{~m} / \mathrm{min}$. The character of the knot decrease was controlled by the optical method. This method allowed to estimate the knot diameter, observed before the microwire fracture.

The bulk magnetic characteristics of the microwires were measured employing the vibration magnetometer with the sensitivity $10^{-6} \mathrm{Gcm}^{3}$. This experimental equipment allows to carry out the measurements of the hysteresis loops of the sample under study at room temperature in the range of magnetic field up to $\pm 10 \mathrm{kOe}$.

The near-surface magnetic characteristics of the wires were measured employing the magneto-optical magnetometer, created on the basis of a high-resolution microscope [21]. The microscope provides a magnification up to 1200 . The size of the studied near-surface area of the sample is determined by the size of the slot, located in the image plane of the microscope before the light detector.

All measurements of near-surface characteristics of the microwires were carried out by means of transverse Kerr effect The TKE magnitude, $\delta$, is determined from relationship $\delta=\left(I-I_{0}\right) / I_{0}$, where $I$ and $I_{0}$ are the light intensities, reflected from the magnetized and nonmagnetized simple, respectively. Actually, the dependences $\delta(H) / \delta_{S} \propto M(H) / M_{S}$ were measured, where $\delta_{S}$ is the TKE magnitude at $M=M_{S} ; M_{S}$ is the saturation magnetization of the sample, $H$ is the external remagnetizing magnetic field.

The magneto-optical Kerr effects are known to be sensitive to the magnetization of the near-surface layer of a certain thickness, corresponding to light penetration depth into medium $t_{\mathrm{pen}}$. The value of $t_{\mathrm{pen}}$ is determined from relationship $t_{\mathrm{pen}}=\lambda / 4 \pi k$, where $\lambda$ is the wavelength of the incident light, and $k$ is the absorption coefficient of the medium. According to the existing experimental data [22], the values of $t_{\mathrm{pen}}$ of magnetic materials in the energy range of incident light photons of $0.5-6.0 \mathrm{eV}$ do not exceed 10-30 nm. In our case, the near-surface layer thickness under study was $20 \mathrm{~nm}$.

The simples under study had the length of $20 \mathrm{~mm}$. The external remagnetizing magnetic field was applied parallel to the microwire length, $L$.

The near-surface hysteresis loops were measured at registration of magneto-optical signals from near-surface areas of $1 \times 0.08 \mathrm{~mm}^{2}$ (the large size is parallel to the microwire length, $L$ ). The external magnetic field was changed from $+H$ to $-H$ and from $-H$ to $+H$. The value of $H$ was significantly larger than $H_{\mathrm{S}}$, where $H_{\mathrm{S}}$ is the saturation field of the sample under study.

The measurements of the local magnetization curves for different near-surface areas and the distributions of the magnetization components along the microwire length $L$, were carried out at registration of the magneto-optical signals from near-surface areas of $2 \times 1 \mu \mathrm{m}^{2}$. These data allow to estimate degree of uniformity of the near-surface magnetic characteristics of the microwires under study.
All the measurements were carried out on the central part of the samples in order to exclude the influence of end effects, in particular, to reduce the influence of variations in the local demagnetizing factors.

\section{Results and Discussion}

According to XRD data, the thick microwires under study retain amorphous structure up to the magnetic core diameter of 90 microns.

The pulling rate of the microwires was found to be nearly two orders lower than the rate used in other methods of producing microwires with the same cross sections. The glass shell in such wires is weakly adhered with the metallic core and can be easily removed.

The wire cores have the stable geometric parameters along their lengths, the smooth (almost without defects) mirror surfaces and high tensile strength on stretching of $3000 \mathrm{MPa}$ [23].

The thick microwires were found to exhibit very high plasticity level, which is characterized by its ability to be tied into a knot without fracture (Figures 1).

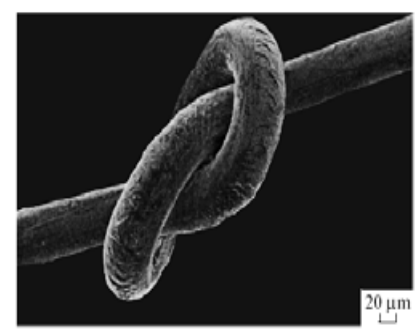

Figure 1. Image of the thick $\mathrm{Co}_{69} \mathrm{Fe}_{4} \mathrm{Cr}_{4} \mathrm{Si}_{12} \mathrm{~B}_{11}$ microwires, illustrating its ability to tighten into a knot without fracture.

The type of fractures of such wires after tests for stretching is venous. However, the fracture of the as-cast thick wire consists of one or several pronounced main veins (Figure 2A). Such character of the vein formation testifies about very high microplasticity of the thick wires in the fracture zone.

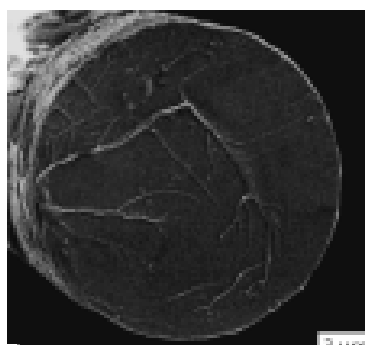

A

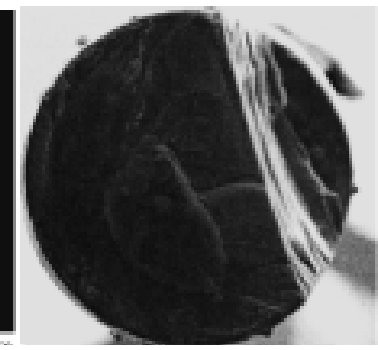

B
Figure 2. Fractographies of the fracture surface, observed for the as-cast and annealed at $\mathrm{T}=430{ }^{\circ} \mathrm{C}$ thick $\mathrm{Co}_{69} \mathrm{Fe}_{4} \mathrm{Cr}_{4} \mathrm{Si}_{12} \mathrm{~B}_{11}$ microwire: $\mathrm{A}$ and $\mathrm{B}$, correspondingly.

It was found that annealing of the $\mathrm{Co}_{69} \mathrm{Fe}_{4} \mathrm{Cr}_{4} \mathrm{Si}_{12} \mathrm{~B}_{11}$ microwire at temperatures up to $300^{\circ} \mathrm{C}$ does not change the mechanical properties and fractograpfic pattern of the fracture. Annealing at $T>350^{\circ} \mathrm{C}$ is accompanied by 
increasing embrittlement that leads to a decrease in tensile strength and plasticity. After annealing at $T=430^{\circ} \mathrm{C}$, the brittle fracture of the alloy takes place. In this case, the fracture consists of two parts: one part of the wire is fractured at the shear band perpendicularly to the longitudinal wire axis, and other part is fractured at the small angle, forming a deep wavy pit in the opposite side (Figure 2B). No crystalline phase was observed in both fracture parts.

The hysteresis loops for the initial and annealed samples were measured employing the magneto-optical and vibration magnetometers. Figure 3 (A-D) shows the hysteresis loops, received for the as-cast and annealed at various temperatures $\mathrm{Co}_{69} \mathrm{Fe}_{4} \mathrm{Cr}_{4} \mathrm{Si}_{12} \mathrm{~B}_{11}$ samples employing the magneto-optical magnetometer. Figure 4 shows the most characteristic hysteresis loops, received by mean of the vibration magnetometer.

These data allowed to obtain the dependences of the saturation field $H_{S}$ and coercive force $H_{C}$ on the annealing temperature. Analysis of experimental data showed the following. The marked increases of the near-surface magnitudes of the saturation field and the coercivity are observed at $T \geq 300^{\circ} \mathrm{C}$ (Figure 5A). The magnitude of $H_{C}{ }^{\mathrm{BULK}}$ (also as $H_{S}^{\mathrm{BULK}}$ ) for the samples, annealed at $T \leq$ $400^{\circ} \mathrm{C}$, are almost unchanged (Figure $5 \mathrm{~B}$ ). At $T=430^{\circ} \mathrm{C}$, as the near-surface and bulk values of the coercivity increase, and the shape of the near-surface hysteresis loop is changed (Figure 3D).

Moreover, from Figure 5 one can see that the values of $H_{C}{ }^{S U R}$ are higher than $H_{C}{ }^{B U L K}$. Analogous difference was found for $H_{S}^{S U R}$ and $H_{S}^{B U L K}$.
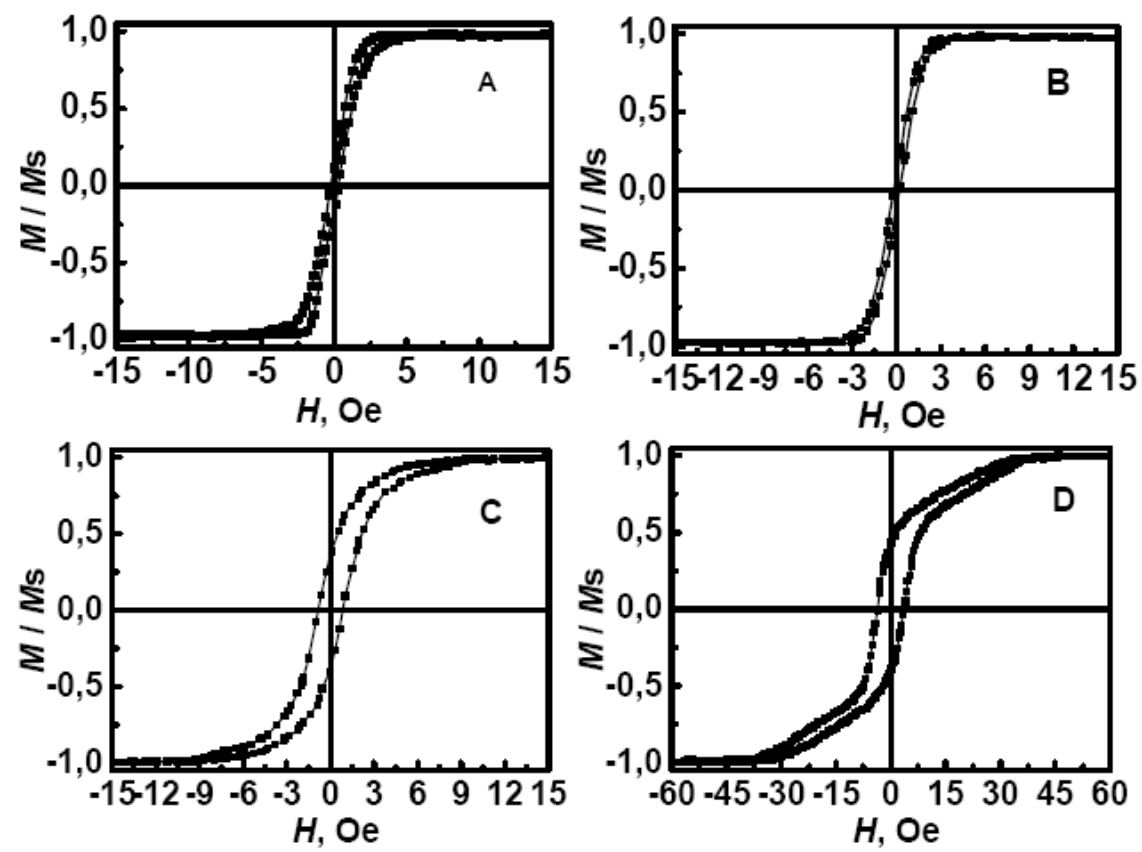

Figure 3. Near-surface hysteresis loops, obtained employing the magneto-optical magnetometer for the as-cast $\mathrm{Co}_{69} \mathrm{Fe}_{4} \mathrm{Cr}_{4} \mathrm{Si}_{12} \mathrm{~B}_{11}$ microwire $(\mathrm{A})$ and the microwires, annealed at $T=200(\mathrm{~B}), 350$ (C) and $430^{\circ} \mathrm{C}(\mathrm{D})$.
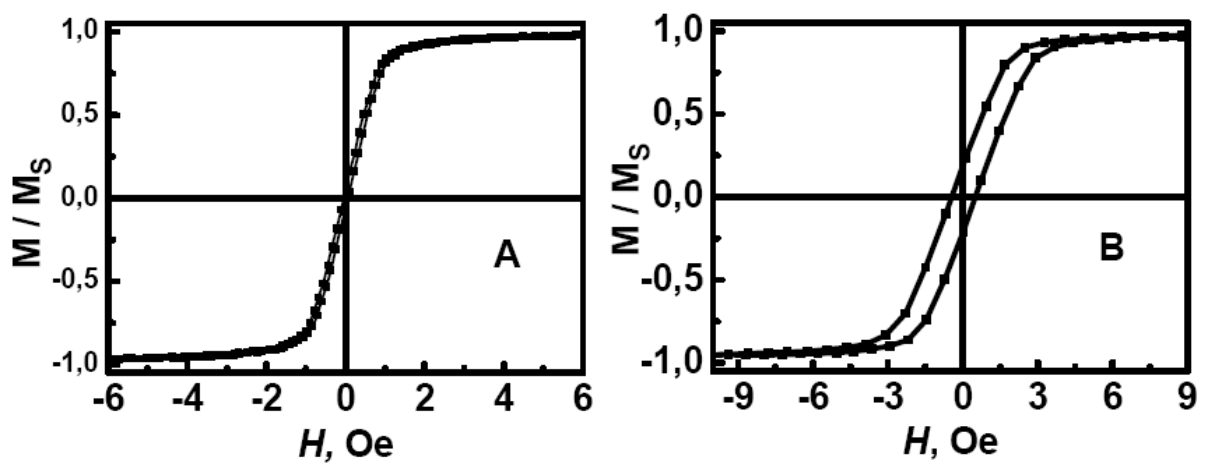

Figure 4. Hysteresis loops, obtained employing vibration magnetometer for the $\mathrm{Co}_{69} \mathrm{Fe}_{4} \mathrm{Cr}_{4} \mathrm{Si}_{12} \mathrm{~B}_{11}$ microwire, annealed at $T=200{ }^{\circ} \mathrm{C}(\mathrm{A})$ and $430{ }^{\circ} \mathrm{C}(\mathrm{B})$, 

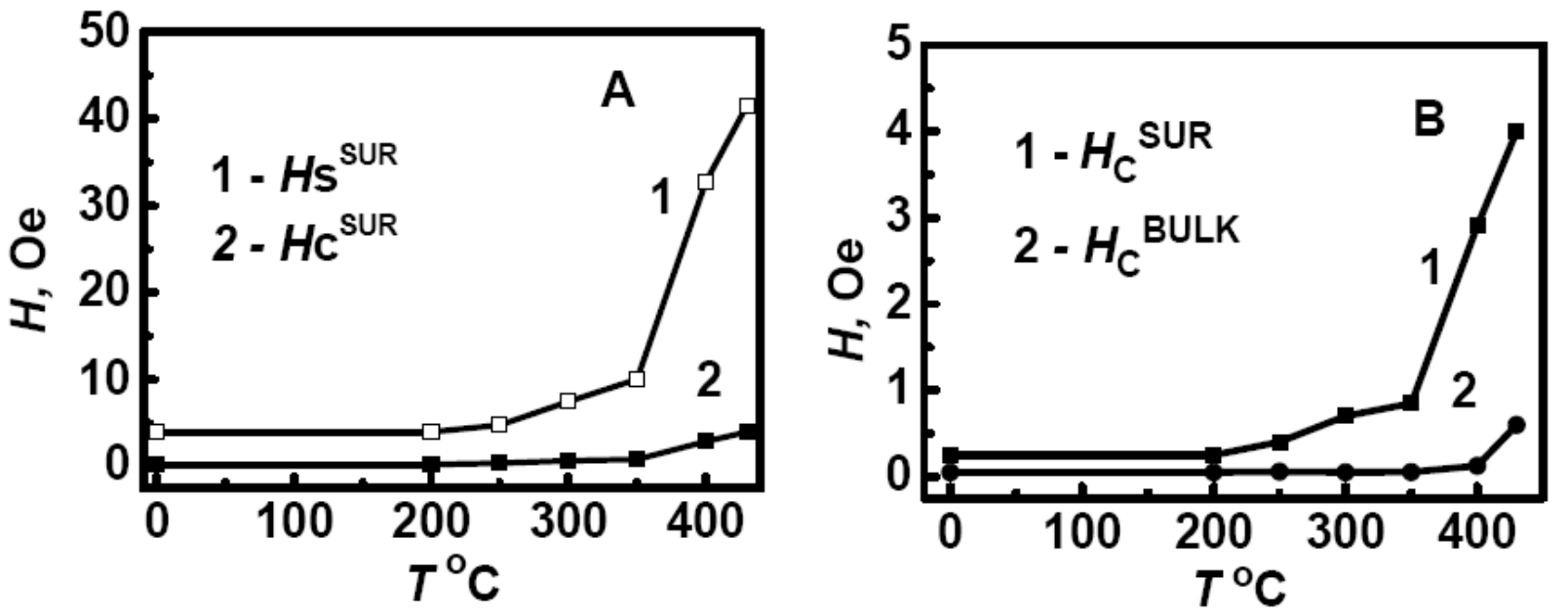

Figure 5. Dependences of the near-surface values of the saturation field and the coercivity on the annealing temperature and the temperature dependences of the near-surface and bulk values of the coercivity, obtained for the $\mathrm{Co}_{69} \mathrm{Fe}_{4} \mathrm{Cr}_{4} \mathrm{Si}_{12} \mathrm{~B}_{11}$ microwire: (A) and (B), correspondingly.
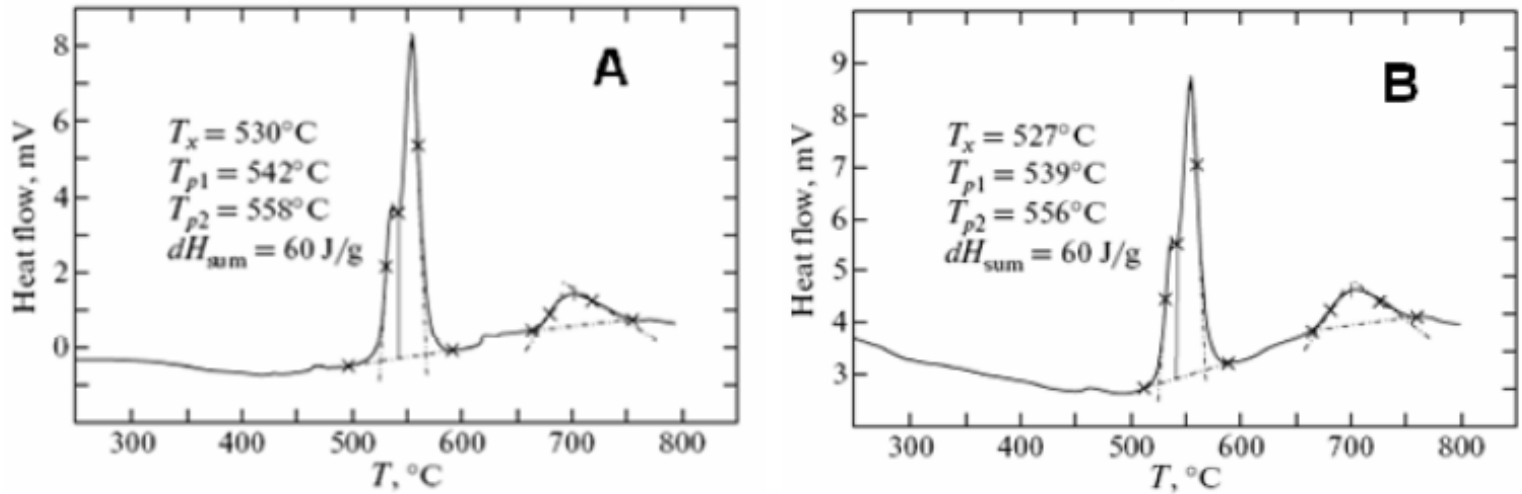

Figure 6. DSC thermograms, observed for the as-cast $\mathrm{Co}_{69} \mathrm{Fe}_{4} \mathrm{Cr}_{4} \mathrm{Si}_{12} \mathrm{~B}_{11}$ microwire and the microwire, annealed at $T=430^{\circ} \mathrm{C}$ : (A) and (B), correspondingly. $T x$ is the temperature of the crystallization onset; $T p 1$ and $T p 2$ are the temperatures of the peaks of the crystallization stages; $d H s u m$ is the total thermal effect of two crystallization stages

The experimental data can be explained by the structural peculiarities of the microwires, in particular, using data of differential scanning calorimetry (DSC), received for the samples under study (Figure 6). From Figure 6 one can see that the temperatures of crystallization onset, $T \mathrm{x}$, of the as-cast and the annealed at $T=430^{\circ} \mathrm{C}$ of the thick microwires are almost identical. This fact shows that amorphous structure in the wire volume remains completely after annealing at temperatures up to $430^{\circ} \mathrm{C}$. As a result, the bulk values of the coercivity and the saturation field depend weakly on temperature.

At the same time, the magneto-optical investigations showed that the near-surface values of the saturation field and the coercivity of the annealed microwires change even at $T \geq 300^{\circ} \mathrm{C}$. It should be noted that this result agrees with our magneto-optical studies of the annealed amorphous ribbons [24]. It was found in [24] that main changes of the magnetic properties of the annealed ribbons are observed in the near-surface layer. The thickness of this layer is about 1 micron. Moreover, it was revealed in [24] that after etching of the near-surface layer up to the above noted thickness, the magnetic properties of the etched ribbons coincide with the characteristics of the as-cast ribbons within the limit of experimental error.

The more complicated near-surface hysteresis loop, observed for the microwire annealed at $T=430^{\circ} \mathrm{C}$ (Fig. 3D), testifies the appearance of two-phase magnetic structure in the near-surface layer. The above near-surface hysteresis loop, and also the increase of the magnitudes of the magnetic characteristics can be explained by the development of nanocrystallization process in the near-surface layer. It should be noted that similar effect of the near-surface nanocrystallization in amorphous $\mathrm{Fe}-$ rich and $\mathrm{Co}-$ rich microwires was also experimentally observed in [25].

The difference between the bulk and near-surface values of $H_{S}$ and $H_{C}$, increasing at $T \geq 300^{\circ} \mathrm{C}$, can be explained by the existence of chemical and microstructural heterogeneities on the microwire surface that are usually observed in amorphous materials $[24,26]$.

To obtain complete information on the properties of the microwires, the magnetic characteristics of the as-cast sample with removed mechanically glass shell were also measured. It was found that the near-surface and the bulk magnitudes of the magnetic characteristics of the microwires 
with a glass shell and without it coincide within the limit of experimental error (see Table 1). Similar measurements were also performed on the $\mathrm{Co}_{69} \mathrm{Fe}_{4} \mathrm{Cr}_{4} \mathrm{Si}_{12} \mathrm{~B}_{11}$ microwires with the other diameters.

It was found that the magnetic characteristics of these microwires after removal of glass shell do not change also. For illustration of this fact, the results of the measurements of the magnetic characteristics of the microwire with the diameter of $d=75$ microns are presented also in Table 1 .

This property of the thick microwires can be explained by the following. As it was noted above, the modernized Ulitovsky-Taylor method, used for fabricating the thick microwires, does not provide complete adhesion of the metallic core with the glass shell. As a result, the stresses, induced by the interaction of the magnetic core with the glass shell, do not influence on the magnetic-field behavior of the microwires.

At last, the measurements of the local magnetization curves and the distributions of the magnetization components, parallel to the microwire length, were carried out employing the magneto-optical method. The experimentally found dependences $\delta(H) / \delta s \propto M(H) / M s$ at changing $H$ from 0 to $H>H s$ and $\delta(L) / \delta s \propto M(L) / M s$ at the fixes magnitudes of $H$ are presented in Figures 7 and 8, correspondingly.

Table 1. Magnetic characteristics of the microwires with a glass shell and without it

\begin{tabular}{|c|c|c|}
\hline $\mathrm{d} / \mathrm{D}, \mu \mathrm{m}$ & $H_{\mathrm{C}}{ }^{\text {BULK }}$, Oe & $H_{\mathrm{C}}{ }^{\text {SUR }}$, Oe \\
\hline $90 / 125$ & 0.05 & 0.2 \\
\hline $90 /$ without glass & 0.05 & 0.2 \\
\hline $75 / 125$ & 0.06 & 0.25 \\
\hline $75 /$ without glass & 0.06 & 0.25 \\
\hline
\end{tabular}
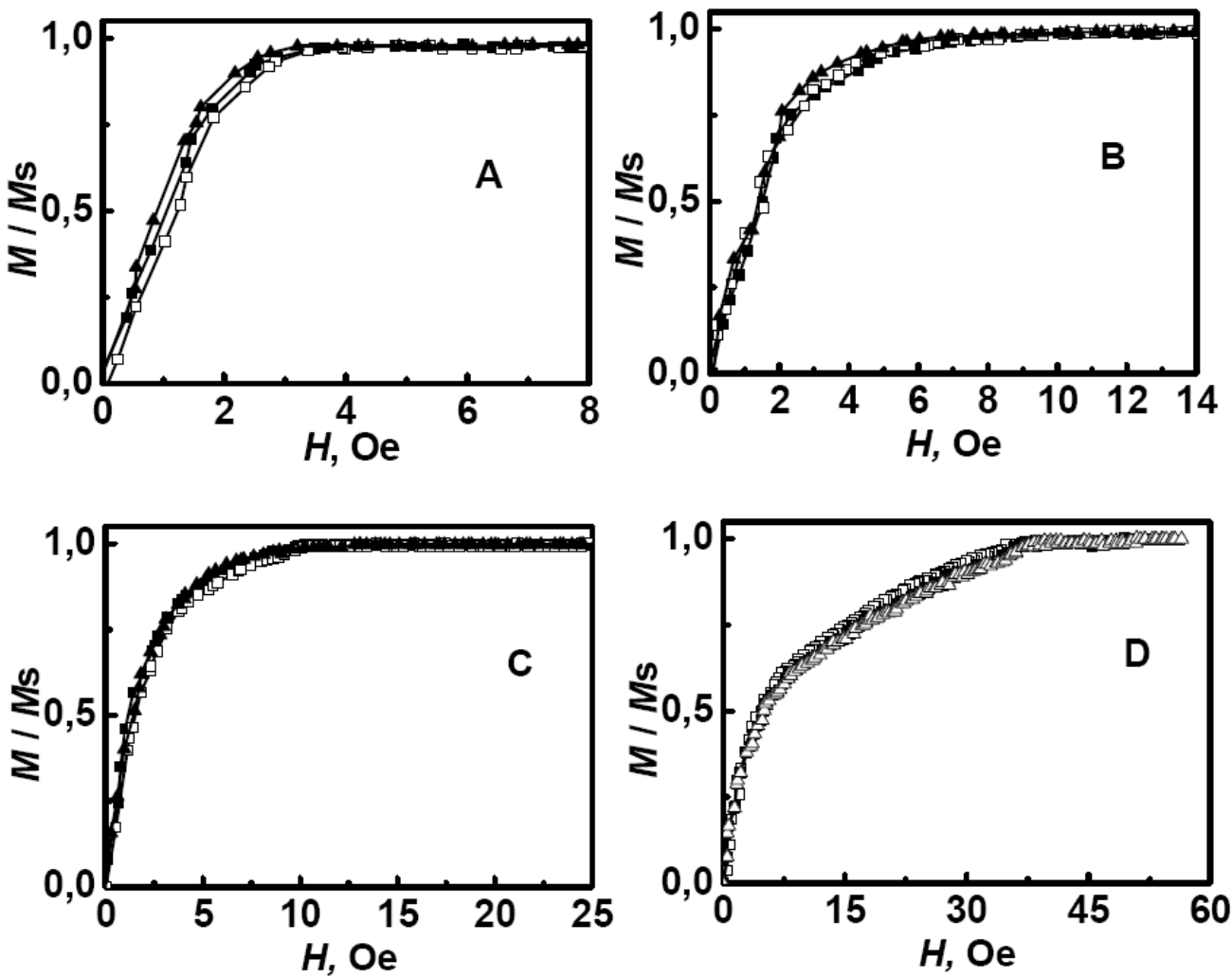

Figure 7. Near-surface local magnetization curves, observed for different near-surface areas of the $\mathrm{Co}_{69} \mathrm{Fe}_{4} \mathrm{Cr}_{4} \mathrm{Si}_{12} \mathrm{~B}_{11}$ microwire, annealed at $T=200(\mathrm{~A})$, 300 (B), 350 (C) and $430 \mathrm{C}(\mathrm{D})$. 

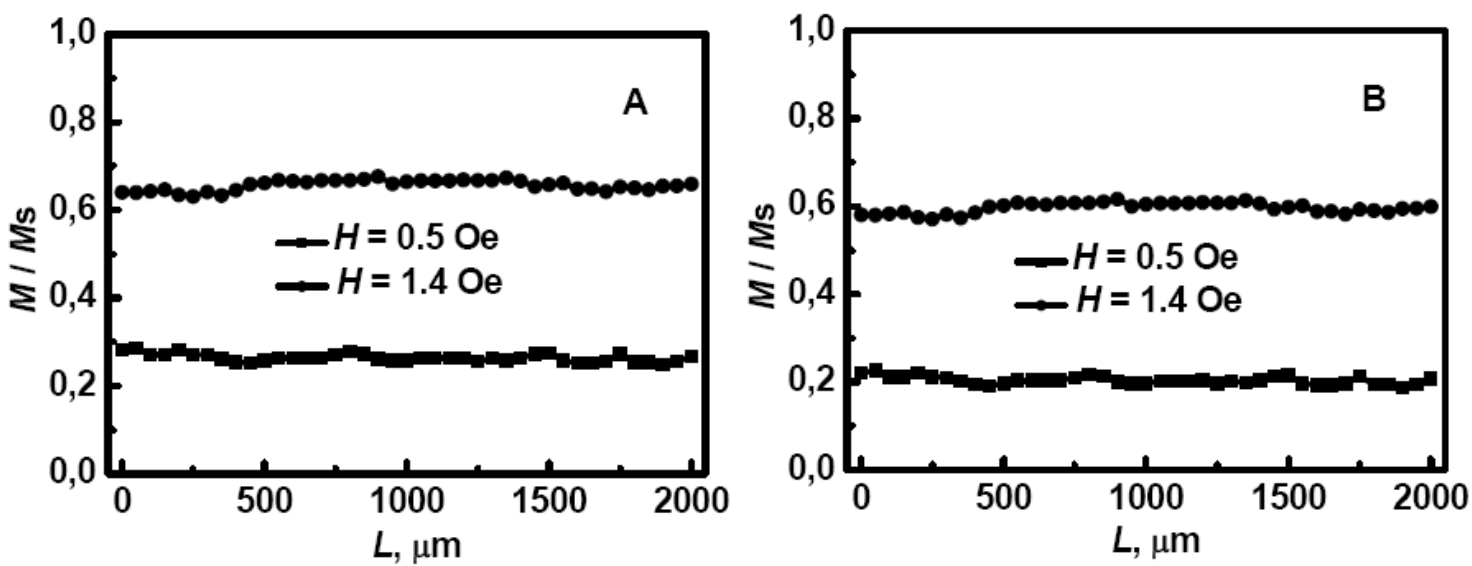

Figure 8. Distributions of near-surface magnetization components along to the microwire length $L$, observed for the $\mathrm{Co}_{69} \mathrm{Fe}_{4} \mathrm{Cr}_{4} \mathrm{Si}_{12} \mathrm{~B}_{11}$ microwires, annealed at $T=200^{\circ} \mathrm{C}(\mathrm{A})$ and $350^{\circ} \mathrm{C}(\mathrm{B})$.

Analysis of these data showed that the $\mathrm{Co}_{69} \mathrm{Fe}_{4} \mathrm{Cr}_{4} \mathrm{Si}_{12} \mathrm{~B}_{11}$ microwires exhibit the negligible distinctions of the local near-surface magnetization curves (Figure 7) and local magnetization magnitudes of $M / M \mathrm{~s}$ (Figure 8). It should be noted that the local hysteresis loops were revealed to have analogous behavior.

It was found that the variations of the near-surface local magnitudes of the saturation field and $M / M \mathrm{~s}$ of the as-cast and annealed microwires don't exceed $8 \%$. These results are evidence of the high homogeneity of the local magnetic properties of the wires, which can be ascribed to the slight dispersion of magnetic anisotropy, which is in turn caused by the stable geometric parameters of the microwires along their lengths.

\section{Conclusion}

The results of the study of the structural and magnetic properties of the thick as-cast and annealed $\mathrm{Co}_{69} \mathrm{Fe}_{4} \mathrm{Cr}_{4} \mathrm{Si}_{12} \mathrm{~B}_{11}$ microwires allow to draw the following conclusions.

(1) The microwire cores have the stable geometric parameters along their lengths and the smooth (almost without defects) surfaces.

(2) The thick amorphous microwires are characterized by the high plasticity and the high strength.

(3) Destruction of a microwire doesn't happen even after a full tightening it in knot.

(4) The $\mathrm{Co}_{69} \mathrm{Fe}_{4} \mathrm{Cr}_{4} \mathrm{Si}_{12} \mathrm{~B}_{11}$ microwires exhibit the unique magnetic properties. In particular, the volume magnitudes of coercivity of the thick microwires do not exceed 1 Oe.

(5) Heat treatment of the thick $\mathrm{Co}_{69} \mathrm{Fe}_{4} \mathrm{Cr}_{4} \mathrm{Si}_{12} \mathrm{~B}_{11}$ microwires at $T<300^{\circ} \mathrm{C}$ does not influence practically on the magnitudes of the coercivity and the saturation field.

(6) Annealing of the thick microwires at $T=430^{\circ} \mathrm{C}$ causes nanocrystallization process in the near-surface layer of the microwire. As a result, the near-surface magnitudes of $H_{S}$ and $H_{C}$ increase in comparison with the as-cast sample, and the near-surface hysteresis loop has the complicated shape.

(7) The stable geometric parameters of the $\mathrm{Co}_{69} \mathrm{Fe}_{4} \mathrm{Cr}_{4} \mathrm{Si}_{12} \mathrm{~B}_{11}$ microwires along their lengths cause the slight dispersion of magnetic anisotropy of the near-surface layers. As a result, the thick microwires are characterized the high homogeneity of the near-surface local magnetic properties

(8) The discovered properties of the thick $\mathrm{Co}_{69} \mathrm{Fe}_{4} \mathrm{Cr}_{4} \mathrm{Si}_{12} \mathrm{~B}_{11}$ amorphous microwire make these materials promising for practical applications. In particular, thick high-strength Co-rich microwires can find applications at manufacturing of constructional and functional materials, and also composites, containing microwires as a power element.

\section{REFERENCES}

[1] K. Suzuki, H. Fujimori, K. Hashimoto. Amorphous Metals, Butterworths, London, 1982; Metallurgy, Moscow, p.328, 1987 (in Russian).

[2] K. Handrich, S. Kobe. Amorphe Ferro- and Ferrimagnetika, Akademie, Berlin, 1980; Mir, Moscow, p.296, 1982 (in Russian).

[3] R. Hasegava. Present Status of amorphous soft magnetic alloys, Journal of Magnetism and Magnetic Materials, Vol.215-216, 240-245, 2000.

[4] A.F. Cobeno, A.P. Zhukov, E. Pina, J.M. Blanco, J. Gonzalez, J.M. Barandiaran. Sensitive Magnetoelastic properties of amorphous ribbon for Magnetoelastic sensors, Journal of Magnetism and Magnetic Materials, Vol.215-216, 743-745, 2000 .

[5] V. Zhukova, M. Ipatov, A. Zhukov. Thin magnetically soft wires for magnetic microsensors, J. Sensors, Vol.9, 9216-9240, 2006.

[6] M.Z. Kozak, E. Misiuk, W. Kwiatkowski. A converter-type 
magnetometer using amorphous ribbon or wire, J. Applied Physics, Vol.69, 5023-5024, 1991.

[7] K. Inuzuka, K. Mohri. Detection of secondary current and torque of squirrel cage induction motor using magnetic sensor, J. IEEE Transactions on Magnetics, Vol.28, 2178-2180, 1992.

[8] Y. Kashiwagi, T. Kondo, K. Mitsui, K. Mohri. 300 A current sensor using amorphous wire core, J. IEEE Transactions on Magnetics, Vol.26, 1566-1568, 1990.

[9] E. Pulido, R.P.del Real, F. Conde, G. Rivero, M. Vazquez, E. Ascasibar, A. Hernando. Amorphous wire magnetic field and DC current sensor based on the inverse Wiedemann effect, J. IEEE Transactions on Magnetics, Vol.27, 5241-5243, 1991.

[10] K. Mohri, T. Koshzawa, K. Kawashima, H. Yoshida, L.V. Panina. Magneto-inductive effect (MI Effect) in amorphous wires, J. IEEE Transactions on Magnetics, Vol.28, 3150-3152, 1992.

[11] M. Vazquez, A. Hernando. A soft magnetic wire for sensor application, Journal of Magnetism and Magnetic Materials, Vol.30, 939-949, 1996.

[12] P.T. Squire, D. Atkinson, M.R.J. Gibbs, S. Atalay. Amorphous wires and their applications, Journal of Magnetism and Magnetic Materials, Vol.132, 10-21, 1994.

[13] K. Bushida, K. Mohri, T. Uchiyama. Sensitive and quick response micro magnetic sensor using amorphous wire $\mathrm{MI}$ element colpitts oscillator, J. IEEE Transactions on Magnetics, Vol.31, Issue 6, Part 1, 3134-3136, 1995.

[14] L. Panina, K. D. Makhnovskiy, K. Mohri. Magnetoimpedance in amorphous wires and multifunctional applications: from sensors to tunable artificial microwave materials, Journal of Magnetism and Magnetic Materials, Vol. 272-276, 1452-1461, 2004.

[15] Y. Abe, K. Miyazawa, M. Nakamura, T. Ohashi. Behavior of metal jet in the in-rotating-water spinning method, Transactions of the Iron and Steel Institute of Japan, Vol.27, N.12, 929-935, 1987.

[16] G.F. Taylor. A method of drawing metallic filaments and a discussion of their properties and uses, J. Physical Review, Vol.23, Issue 5, 655-660, 1924.
[17] A.V. Ulitovski, I.M. Maianski, A.I. Avramenco. Method of continuous casting of glass coated microwire. Patent No 128427, USSR, 15.05.60. Bulletin. No 10, P.14, 1960.

[18] A.V. Ulitovski, N.M. Avernin. Method of fabrication of metallic microwire, Patent No 161325, USSR, 19.03.64. Bulletin No7, P.14, 1964.

[19] P.P. Umnov, V.V. Molokanov, Yu. S. Shalimov, N.V. Umnova, T.R. Chueva, V.T. Zabolotnyi. Peculiarities of obtaining of amorphous wire by Ulitovsky-Taylor method with using continuous process of moulding, Journal of Perspective materials, Vol.2, 87-91, 2010 (in Russian).

[20] E.E. Shalygina, N.V/ Umnova, P.P Umnov, V.V. Molokanov, V.V. Samsonova, A.N. Shalygin, A.A. Rozhnovskaya. Specific Features of magnetic properties of "thick" microwires produced by the Ulitovsky-Taylor method, J. Physics of the Solid State, Vol. 54, No 2, 287-292, 2012.

[21] E.E. Shalygina, M.A. Komarova, V.V. Molokanov. Magneto-optical investigation of Co-rich amorphous microwires, Journal of Experimental and Theoretical Physics, Vol.95, No.3, 511-516, 2002.

[22] E.E. Shalyguina, K.H. Shin. Influence of nonmagnetic layer (Ti, Zr, Pt) on magnetic and Magneto- optical properties of $\mathrm{Fe} / \mathrm{NML}$ bilayers and $\mathrm{Fe} / \mathrm{NML} / \mathrm{Fe}$ trilayers, Journal of Magnetism and Magnetic Materials, Vol.220, 167-174, 2000.

[23] I.I. Mokhirev, T.R. Chueva, V.T. Zabolotnyi, P.P. Umnov, N.V. Umnova, V.V. Molokanov. Strength and plastic properties of amorphous Co-rich wire, obtained by using different methods of fast training of fusion, J. Deformation and Destruction, Vol.7, 31-36, 2010.

[24] E.E. Shalyguina, E.A. Ganshina, Y.W. Rheem, Chong-Oh Kim, CheolGi Kim. Near-surface properties of Co-based amorphous ribbons, J. Physica B, Vol.327, 300-303, 2003.

[25] G.E. Abrosimova, A.S. Aronin, N.N. Holstinina. The influence of glass shall on crystallization Fe-rich and Co-rich microwires, J. Physics of Metals and Metallurgical Science, Vol. 110, No.1, 38-44, 2010.

[26] E.E. Shalyguina, M.A. Komarova, V.V. Molokanov, Chong-Oh Kim, CheolGi Kim, Y.W. Rheem. Near-surface magnetic properties and giant magnetoimpedance of Co-based amorphous ribbons, Journal of Magnetism and Magnetic Materials, Vol.258-259, 174-176, 2003. 